\begin{tabular}{l|l|l|}
\cline { 3 - 3 } CRITICAL & $\begin{array}{l}\text { Western Ghats } \\
\text { EARTNERSHIP FUND }\end{array}$ \\
Special Series
\end{tabular}

\title{
FISHES OF RIVER BHARATHAPUZHA, KERALA, INDIA: DIVERSITY, DISTRIBUTION, THREATS AND CONSERVATION
}

\author{
A. Bijukumar ${ }^{1}$, Siby Philip ${ }^{2}$, Anvar $\mathrm{Ali}^{3}$, S. Sushama ${ }^{4}$ \& Rajeev Raghavan ${ }^{5}$ \\ ${ }^{1}$ Department of Aquatic Biology and Fisheries, University of Kerala, Thiruvananthapuram, Kerala 695581, India \\ ${ }^{2}$ Department of Zoology, Nirmalagiri College, Koothuparamba, Kerala 670701, India \\ 2,3,5 Conservation Research Group (CRG), St. Albert's College, Kochi, Kerala 682018, India \\ ${ }^{4}$ Department of Zoology, N.S.S. College, Ottapalam, Kerala 679103, India \\ ${ }^{5}$ Zoo Outreach Organization (ZOO), 96, Kumudham Nagar, Vilankurichi Road, Coimbatore, Tamil Nadu 641035, India \\ 19abiju@rediffmail.com, ${ }^{2}$ siby@conservationresearchgroup.org, ${ }^{3}$ anvaraliif@gmail.com, ${ }^{4}$ sumakrishnan3@yahoo.com, \\ ${ }^{5}$ rajeevraq@hotmail.com (corresponding author)
}

\begin{abstract}
We present here a comprehensive account of the diversity, distribution, threats, and suggest conservation measures for the fishes of Bharathapuzha, the largest west flowing river in the southern Indian state of Kerala. A total of 117 species under 43 families and 81 genera were recorded from the river, of which 98 were primary freshwater and 19 were secondary freshwater and/or diadromous species. Six species of non-native fish were also recorded, of which three were exotic to the country and three were transplanted from the gangetic plains. Twenty-eight percent $(S=33)$ of species that occur in the Bharathapuzha are endemic to the Western Ghats, while three species (Balitora jalpalli, Mesonoemacheilus remadevii and Pseudolaguvia austrina) are restricted in their distribution to the river system. A little more than one-tenth (11\%; S = 13) of species that occur in the river are listed under various threatened categories on the IUCN Red List. As part of this study, we also extend the distribution range of Osteochilichthys longidorsalis to the Bharathapuzha River system, based on its collection from the Thoothapuzha tributary. Several anthropogenic stressors including deforestation and loss of riparian cover, dams and other impoundments, pollution, sand mining, non-native species and destructive fishing practices are threatening the rich ichthyofaunal diversity and endemism in the Bharathapuzha. There is hence an urgent need to develop and implement conservation plans, some of which are discussed.
\end{abstract}

Keywords: Osteochilichthys longidorsalis, Nila, river conservation, Silent Valley National Park, Western Ghats.

DOI: http://dx.doi.org/10.11609/JoTT.03640.4979-93 | ZooBank: urn:Isid:zoobank.org:pub:724397D3-D300-46AF-9CC2-ABDF419384E2

Editor: Anonymity requested.

Date of publication: 26 November 2013 (online \& print)

Manuscript details: Ms \# 03640 | Received 26 May 2013 | Final received 08 November 2013 | Finally accepted 10 November 2013

Citation: Bijukumar, A., S. Philip, A. Ali, S. Sushama \& R. Raghavan (2013). Fishes of River Bharathapuzha, Kerala, India: diversity, distribution, threats and conservation. Journal of Threatened Taxa 5(15): 4979-4993; http://dx.doi.org/10.11609/JoTT.o3640.4979-93

Copyright: (c) Bijukumar et al. 2013. Creative Commons Attribution 3.0 Unported License. JoTT allows unrestricted use of this article in any medium, reproduction and distribution by providing adequate credit to the authors and the source of publication.

Funding: A. Bijukumar thanks the Department of Science, Engineering and Research Council (SERC), Govt of India (No. SR/FT/L-21/2003) and the University Grants Commission (UGC) (No.F.1.15/97 (MINOR/SRO) for financial assistance. Rajeev Raghavan thanks the Critical Ecosystem Partnership Fund (CEPF) - Western Ghats Program through the Ashoka Trust for Research in Ecology and Environment (ATREE), Bangaluru, India for funding.

Competing Interest: The authors declare no competing interests. Funding sources had no role in study design, data collection, results interpretation and manuscript writing.

Author contributions: $A B, S P, A A, S S \& R R$ carried out the field surveys; $A B$ and RR analyzed and interpreted the data; $A B, S P$ \& RR wrote the manuscript.

Author Details: See end of this article.

Acknowledgements: Field work benefitted from the help and support of Josin Tharian, Fibin Baby, Benno Pereira, K. Krishnakumar, M.R. Ramprasanth, Sanjay Molur and Nibha Namboodiri. Field work in the Silent Valley National Park was conducted with official permits from the Kerala State Forest and Wildlife Department to A. Bijukumar (WL12-3423/2004) and Rajeev Raghavan (WL12-8550/2009). The authors thank two anonymous reviewers and the subject editor for their constructive comments and suggestions that greatly improved the manuscript.
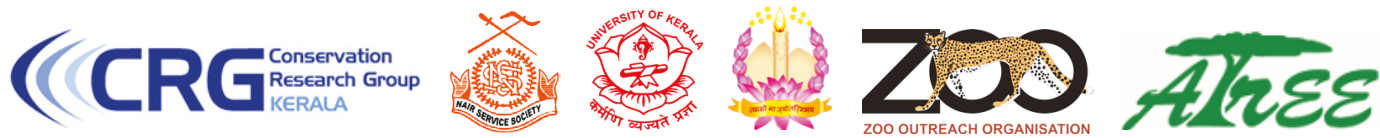

This article forms part of a special series on the Western Ghats of India, disseminating the results of work supported by the Critical Ecosystem Partnership Fund (CEPF), a joint initiative of l'Agence Française de Développement, Conservation International, the European Commission, the Global Environment Facility, the Government of Japan, the MacArthur Foundation and the World Bank. A fundamental goal of CEPF is to ensure civil society is engaged in biodiversity conservation. Implementation of the CEPF investment program in the Western Ghats is led and coordinated by the Ashoka Trust for Research in Ecology and the Environment (ATREE). 


\section{INTRODUCTION}

Information on diversity and distribution of species is crucial for appropriate and timely decision making in biodiversity conservation. Collation and dissemination of such information is especially important for poorly known yet threatened taxa such as freshwater fish, and for critical biodiversity areas such as 'Hotspots'. The Western Ghats part of the Western Ghats - Sri Lanka Biodiversity Hotspot in peninsular India is an exceptional region of freshwater biodiversity (Dahanukar et al. 2011), where in spite of more than 200 years of research the ichthyofauna continues to be influenced by both the Linnaean and Wallacean shortfall (Dahanukar et al. 2011; Raghavan 2011). Although there are hundreds of papers including checklists on freshwater fishes of Kerala, in both peer-reviewed and gray literature, few provide data that can be validated. Most checklists from this region are not supported by voucher specimens, photographs and/or taxonomic notes and are mere compilations of secondary information from some of the earlier 'dated' papers/checklists.

The Bharathapuzha River, also known as 'Nila' and 'Perar', originates from the northern and southern tips of the Palakkad gap in the Western Ghats, as well as from the gap. The minor tributaries join together to form four major tributaries: Gayathripuzha, Chitturpuzha, Kalpathipuzha, and Thoothapuzha (Image 1). It is the second longest $(209 \mathrm{~km})$ and largest (annual discharge of $3.94 \mathrm{~km}^{3}$ ) among the west flowing perennial rivers in the state of Kerala (Raj \& Azeez 2012), as well as the river with the most extensive basin area, second in length and third in yield by thousand million cubic feet (TMCF; Anon 1998). The Bharathapuzha watershed lies between $10^{\circ} 25^{\prime}-11^{\circ} 15^{\prime} \mathrm{N}$ and $75^{\circ} 50^{\prime}-76^{\circ} 55^{\prime} \mathrm{E}$, and is located in the Palakkad, Thrissur and Malappuram districts of Kerala State. Bharathapuzha has a total basin area of $6,186 \mathrm{~km}^{2}$, of which $4,400 \mathrm{~km}^{2}$ is in Kerala and the remaining in Tamil Nadu (Raj \& Azeez 2012).

The earliest ichthyological investigations in the Bharathapuzha drainage (then Ponnani drainage of erstwhile Malabar state in India) were carried out by Jerdon (1849) and Day (1865). This was followed by the works of Herre (1942, 1945), Silas (1951, 1958) and subsequently Indra \& Devi (1981), Devi \& Indra (1984; 1986), Easa \& Basha (1995), Easa \& Shaji (1997), Bijukumar \& Sushama (2001), Shaji (2002), Kurup et al. (2004), Sushama et al. (2004) and Devi et al. (2005). However, most of these studies were restricted to one or a few regions and/or tributaries of the Bharathapuzha, and a comprehensive study has not yet been realized.
Here, we provide a comprehensive and consolidated checklist of fishes of the Bharathapuzha River system (backed by voucher specimens) and discuss their distribution, threats and conservation. We also report on the extension of range of an endemic cyprinid, Osteochilichthys longidorsalis Pethiyagoda \& Kottelat 1994, and a new site record for Pseudolaguvia austrina Radhakrishnan, Kumar \& Ng 2010, in the Bharathapuzha River system.

\section{MATERIALS AND METHODS}

\section{Study area}

Bharathapuzha has four major tributaries, Gayathripuzha, Chitturpuzha (Kannadipuzha or Amaravathipuzha), Kalpathipuzha and Thoothapuzha (Image 1). From the confluence of Kalpathipuzha and Chitturpuzha at Parali, the river acquires the name 'Bharathapuzha'. The flow regime of the river includes highlands $(>76 \mathrm{~m})$, midlands $(76-8 \mathrm{~m})$ and lowlands $(<8 m)$ (Raj \& Azeez 2009). A series of large dams have been constructed across the Bharathapuzha River and its tributaries; two dams are located in Tamil Nadu (Thirumoorthy and Aliyar) and seven in Kerala (Kanjirapuzha, Malampuzha, Walayar, Meenkara, Chulliar, Pothundy and Mangalam). Further, there are two major diversion schemes, Moolathara and Cheerakkuzhy, in addition to a Thrithala-Velliyamkallu regulator-cum-bridge. A series of check dams are built across the lower reaches of Bharathapuzha in order to retain water temporarily.

The Reserved Forest area in the Bharathapuzha Basin in Kerala is around $625 \mathrm{~km}^{2}$, while it is $800 \mathrm{~km}^{2}$ including forest vegetation in Tamil Nadu (Image 2). While Chitturpuzha watershed has forest cover in the Anamalai hills of Tamil Nadu State (Aliyar tributary), the forest patches in Kalpathipuzha, Gayathripuzha and Thoothapuzha are represented by $177 \mathrm{~km}^{2}, 196 \mathrm{~km}^{2}$, and $252 \mathrm{~km}^{2}$ of forest areas respectively in the State of Kerala. The Bharathapuzha and its tributaries also drain three important protected areas, the Indira Gandhi Tiger Reserve, the Parambikulam Tiger Reserve, and the Silent Valley National Park, apart from many areas declared as reserved forests.

\section{Sampling sites and methods}

As part of the present study, surveys were carried out in all the four tributaries, viz., Gayathripuzha, Chitturpuzha, Kalpathipuzha (Image 3) and Thoothapuzha (Image 4) of the Bharathapuzha River and 


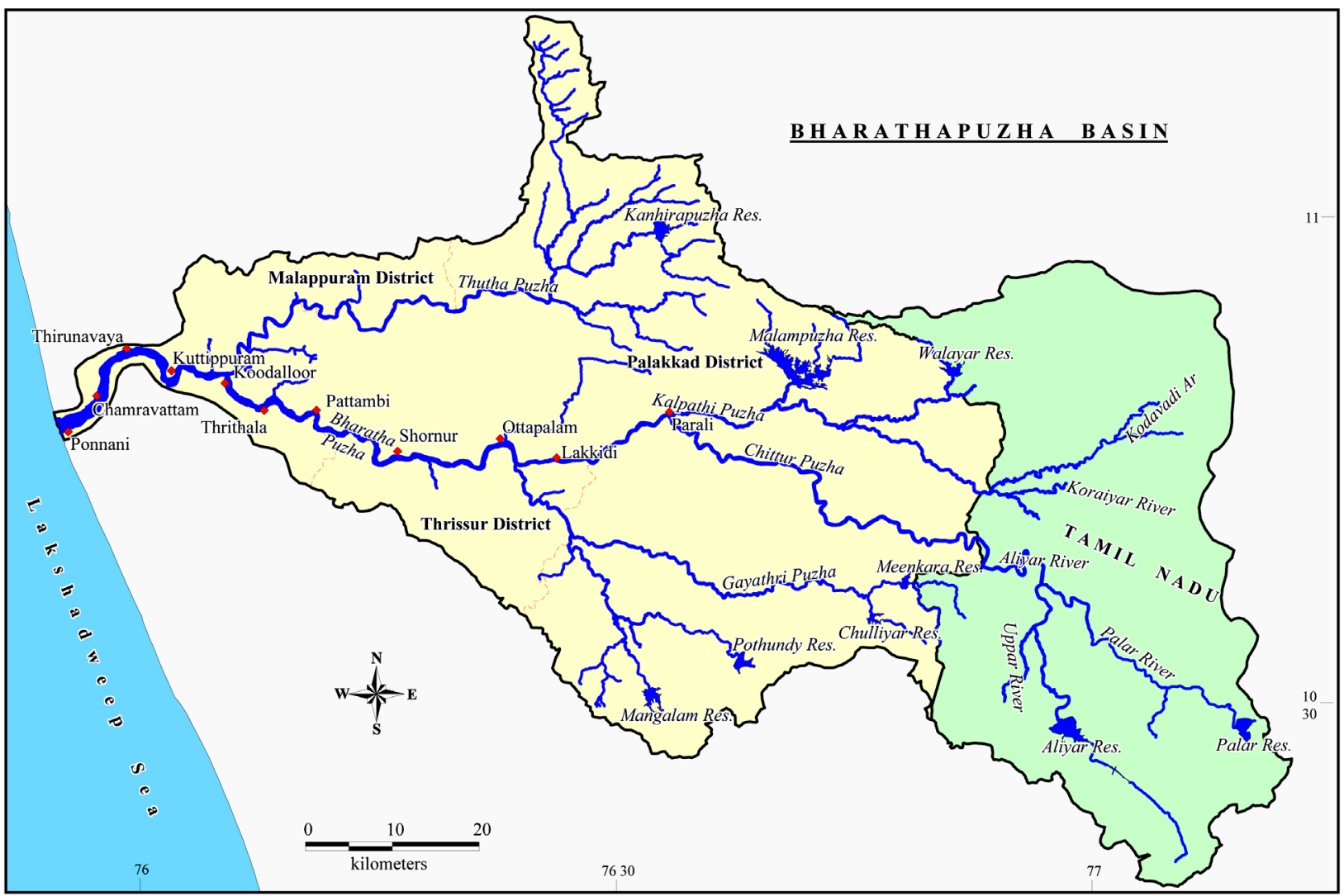

Image 1. Bharathapuzha River basin showing the major tributaries and streams

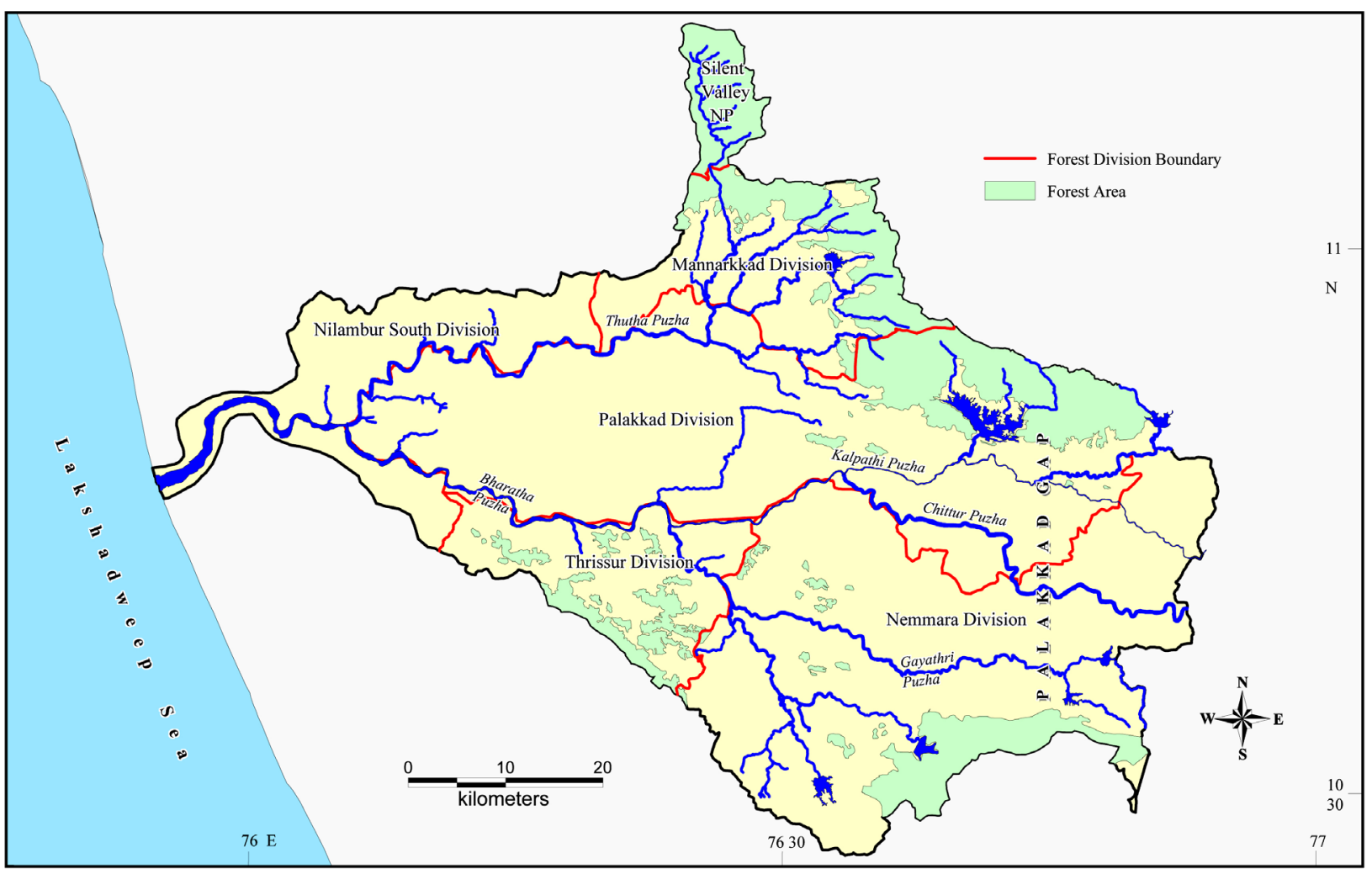

Image 2. Extent of forest cover in the Bharathapuzha River basin 


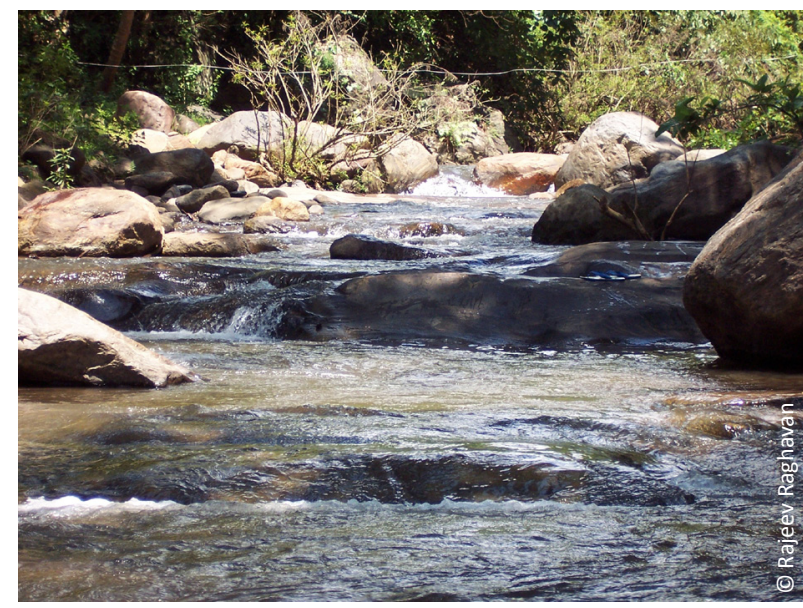

Image 3. Stream in the upper reaches of the Kalpathipuzha tributary of Bharathapuzha River

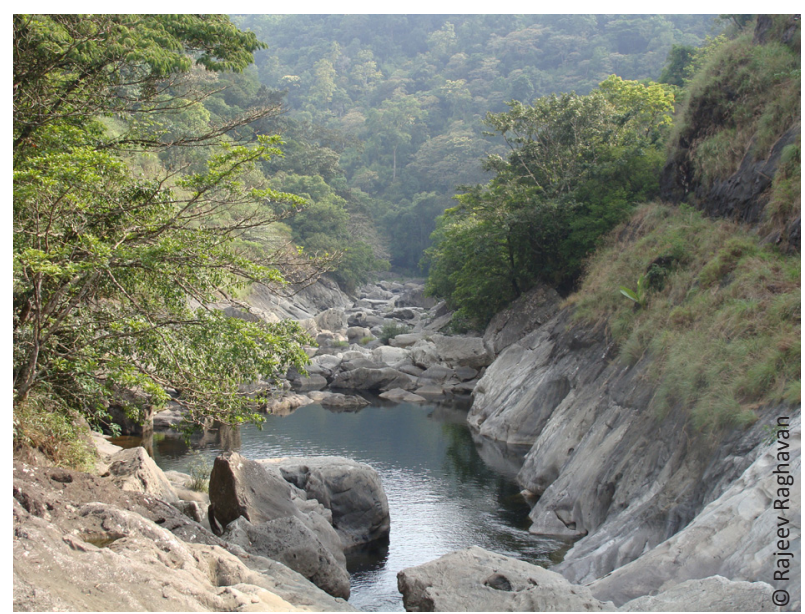

Image 5. Kunthipuzha stream inside the Silent Valley National Park

the Kunthipuzha stream (of Thoothapuzha tributary) flowing through the Silent Valley National Park (Image 5) at multiple intervals from January 2004 to February 2013. Fishes were collected using a variety of active and passive gears such as scoop nets, drag nets, cast nets, gill nets and specially designed and fabricated net made of mosquito nets. Random surveys were also carried out in the major markets and landing centers along all the five tributaries. Voucher specimens were preserved in $4 \%$ formaldehyde and whenever possible tissue samples were preserved in $95 \%$ ethanol, and transferred to the laboratory for further identification.

\section{Species identification and morphometry}

Fishes were identified by comparing measurements and counts of the voucher specimens, with those of the type/type series and/or as mentioned in the original

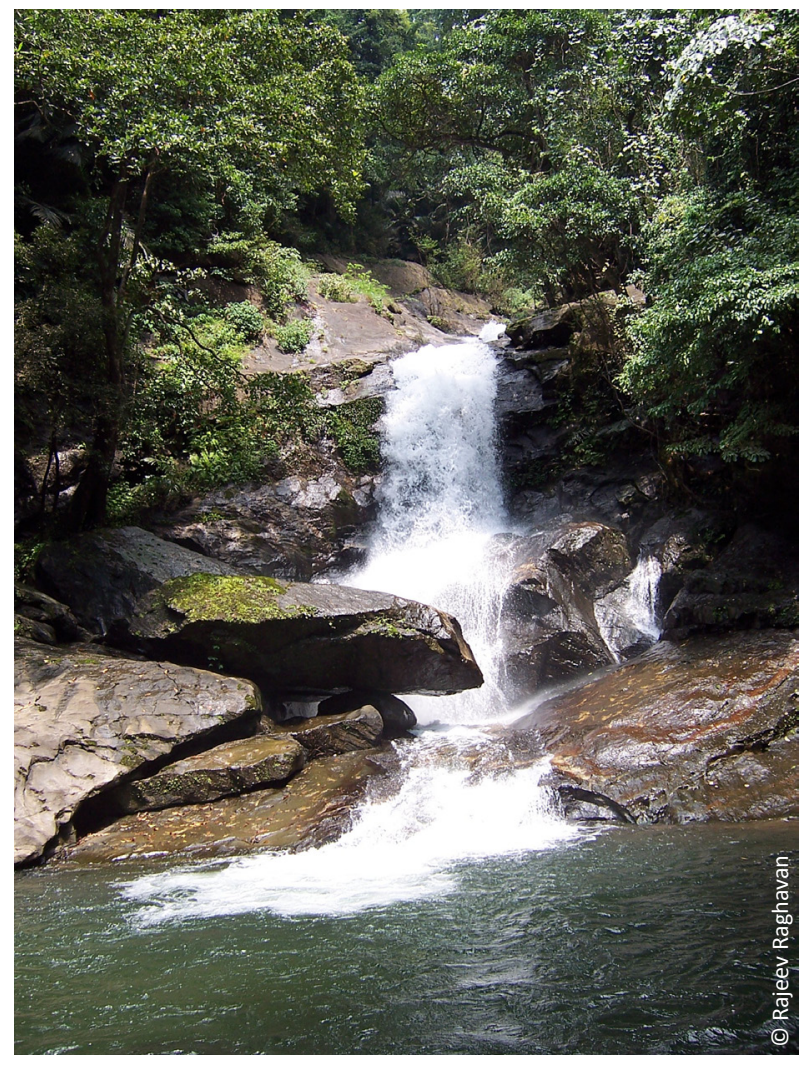

Image 4. A cascade in Meenvallam in the Thoothapuzha tributary of Bharathapuzha River

description. All measurements were taken point to point using dial calipers to the nearest $0.1 \mathrm{~mm}$. Voucher specimens of all species recorded in this paper are deposited at the Museum of the Department of Aquatic Biology and Fisheries, University of Kerala (DAB-UoK), Thiruvananthapuram, Kerala, India and the Conservation Research Group, St. Albert's College (CRG-SAC), Kochi, India. All species names except for the members of the super family Cobitoidea, adhere to the Catalog of Fishes (Eschmeyer 2013) unless otherwise mentioned. For species within the super family Cobitoidea, a recent checklist by Kottelat (2012) has been followed.

\section{RESULTS AND DISCUSSION}

\section{Diversity and distribution}

A total of 117 species under 42 families and 81 genera were recorded from the Bharathapuzha River system (Table 1). Of these, 98 species were primary freshwater, and 19 were secondary freshwater and/ or diadromous species. Six species of non-native fish were also recorded of which three (Cyprinus carpio, Oreochromis mossambicus and $O$. niloticus) are exotic 
to the country; while the rest were the Indian major carps (Catla catla, Cirrhinus mrigala and Labeo rohita) transplanted from the gangetic plains.

The main channel of the Bharathapuzha from Parali to Purathoor estuary had the highest species richness $(S=75)$ followed by the Thoothapuzha $(S=57)$ and Kalpathipuzha ( $S=40$ ) (Fig. 1). Of the 75 species found in the main channel, 19 were secondary freshwater species. Thoothapuzha tributary (excluding the Kunthipuzha stream) has the highest species richness, when only primary freshwater fish species are considered. Although Kunthipuzha stream of the Thoothapuzha tributary flowing through the Silent Valley National Park had the lowest species richness $(S=25)$, it has very high conservation value, as two endemic species, Balitora jalpalli and Mesonoemacheilus remadevii are restricted to this stream.

Thirty-three species (28\%) that occur in the Bharathapuzha River are endemic to the Western Ghats, eight species are endemic to the rivers of Kerala, and three species (Balitora jalpalli, Mesonoemacheilus remadevii and Pseudolaguvia austrina) are endemic to the river system. One more species (listed as Garra sp. in Table 1; see also appendix 1) may be endemic to the Western Ghats, once its taxonomic identity is cleared. All three species endemic to the Bharathapuzha River have a restricted distribution in the Thoothapuzha tributary. While $B$. jalpalli and $M$. remadevii are found in the Kunthipuzha stream, $P$. austrina occurs as small fragmented populations in the Kanjirapuzha and Thoothapuzha streams.

A little more than one-tenth of species (11\%; $S=13$ ) that occur in the Bharathapuzha are listed as threatened in the IUCN Red List of Threatened Species (Fig. 2). This

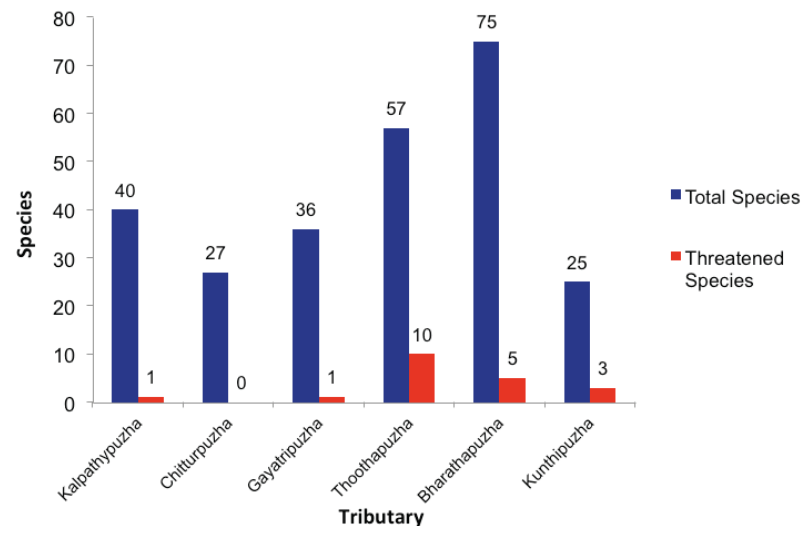

Figure 1. Fish species richness in different tributaries of the Bharathapuzha River and the Kunthipuzha stream in the Silent Valley National Park
Table 1. Fishes of the Bharathapuzha River, their micro-level distribution, endemism and IUCN threat status

\begin{tabular}{|c|c|c|}
\hline Family/Species & Distribution & IUCN Status \\
\hline \multicolumn{3}{|l|}{ Notopteridae } \\
\hline Notopterus notopterus (Pallas, 1769) & S3 & LC \\
\hline \multicolumn{3}{|l|}{ Elopidae } \\
\hline Elops machnata (Forsskål, 1775) & S5 & LC \\
\hline \multicolumn{3}{|l|}{ Megalopidae } \\
\hline $\begin{array}{l}\text { Megalops cyprinoides (Broussonet, } \\
1782)^{5 / D}\end{array}$ & S5 & $\mathrm{DD}$ \\
\hline \multicolumn{3}{|l|}{ Anguillidae } \\
\hline Anguilla bengalensis (Gray, 1831) & S4, S5 & LC \\
\hline A. bicolor McClelland, 1844 & S5 & LC \\
\hline \multicolumn{3}{|l|}{ Clupeidae } \\
\hline Dayella malabarica (Day, 1873)" & S4, S5 & LC \\
\hline Ehirava fluviatilis Deraniyagala, 1929 & S5 & NE \\
\hline \multicolumn{3}{|l|}{ Engraulidae } \\
\hline $\begin{array}{l}\text { Stolephorus commersonii Lacepède, } \\
1803^{5 / D}\end{array}$ & S5 & NE \\
\hline $\begin{array}{l}\text { Thryssa dussumieri (Valenciennes, } \\
1848)^{5 / D}\end{array}$ & S5 & NE \\
\hline \multicolumn{3}{|l|}{ Chanidae } \\
\hline Chanos chanos (Forsskål, 1775) & S5 & NE \\
\hline \multicolumn{3}{|l|}{ Cyprinidae } \\
\hline Catla catla (Hamilton, 1822) & S1, S3, S5 & LC \\
\hline Cirrhinus mrigala (Hamilton, 1822) & S1, S3, S5 & LC \\
\hline Cyprinus carpio Linnaeus, 1758 & S1, S3 & - \\
\hline $\begin{array}{l}\text { Hypselobarbus kurali Menon \& } \\
\text { Remadevi, } 1995^{\text {गा }}\end{array}$ & S1 & LC \\
\hline Labeo fimbriatus (Bloch, 1795) & S1 & LC \\
\hline L. rohita (Hamilton, 1822) & S1, S3, S5 & LC \\
\hline $\begin{array}{l}\text { Osteochilichthys longidorsalis } \\
\text { Pethiyagoda \& Kottelat, 1994" }\end{array}$ & S4 & EN \\
\hline O. nashii (Day, 1869) ตा (Image 11) & s6 & LC \\
\hline $\begin{array}{l}\text { Barbodes carnaticus (Jerdon, 1849) } \\
\text { (Image 12) }\end{array}$ & S6 & LC \\
\hline $\begin{array}{l}\text { Dawkinsia filamentosa } \\
\text { (Valenciennes, 1844) }\end{array}$ & $\mathrm{S} 1, \mathrm{~S} 2, \mathrm{~S} 3, \mathrm{~S} 4, \mathrm{~S} 5$ & LC \\
\hline D. assimilis (Jerdon, 1849) กาก & S4 & VU \\
\hline $\begin{array}{l}\text { Haludaria fasciata (Jerdon, 1849) } \\
\text { (Image 13) }\end{array}$ & S4, S6 & LC \\
\hline Pethia conchonius (Hamilton, 1822) & S2 & LC \\
\hline P. ticto (Hamilton, 1822) & S1, S2, S3, S4, S5 & LC \\
\hline $\begin{array}{l}\text { Puntius amphibius (Valenciennes, } \\
\text { 1842) }\end{array}$ & S1, S3, S4 & $\mathrm{DD}$ \\
\hline P. chola (Hamilton, 1822) & S4, S5 & LC \\
\hline P. mahecola (Valenciennes, 1844) กา & S1, S4 & $\mathrm{DD}$ \\
\hline P. parrah Day, $1865^{\text {กा }}$ & $\mathrm{S} 1, \mathrm{~S} 2, \mathrm{~S} 3, \mathrm{~S} 4, \mathrm{~S} 5$ & LC \\
\hline P. sophore (Hamilton, 1822) & S2 & LC \\
\hline P. vittatus Day, 1865 & S1, S2, S4, S5 & LC \\
\hline Sahyadria denisonii (Day, 1865) กา & S4 & EN \\
\hline Systomus sarana (Hamilton, 1822) & S1, S2, S3, S4, S5 & LC \\
\hline Tor malabaricus (Jerdon, 1849) & S4, S5 & EN \\
\hline
\end{tabular}




\begin{tabular}{|c|c|c|c|c|c|}
\hline Family/Species & Distribution & IUCN Status & Family/Species & Distribution & IUCN Status \\
\hline Laubuca dadiburjori Menon, $1952^{11}$ & S5 & LC & Ompok bimaculatus (Bloch, 1794) & S1, S3, S4 & NT \\
\hline L. fasciata Silas, $1958^{\natural}$ & S4, S6 & VU & O. malabaricus (Valenciennes, 1840) & S1, S4, S5 & LC \\
\hline Salmophasia balookee (Sykes, 1839) & S1, S2, S3, S4 & LC & Schilbeidae & & \\
\hline S. boopis (Day, 1874) ) $^{\text {ก }}$ & S5, S6 & LC & Pseudeutropius mitchelli Günther, & S4 & EN \\
\hline $\begin{array}{l}\text { Amblypharyngodon melettinus } \\
\text { (Valenciennes, 1844) }\end{array}$ & S5 & LC & & & \\
\hline A. microlepis (Bleeker, 1853) & S2, S3, S4, S5 & LC & $\begin{array}{l}\text { Glyptothorax anamalaiensis Silas, } \\
1952^{\text {จा }}\end{array}$ & S4, S5 & EN \\
\hline Barilius bakeri (Day, 1865) ตा & S1, S6 & LC & G. annandalei Hora, 1923 & S4, S6 & LC \\
\hline B. bendelisis (Hamilton, 1807) & S3, S4, S5 & LC & Pseudolaguvia austrina & & \\
\hline B. gatensis (Valenciennes, 1844) กา & S4, S6 & LC & Radhakrishnan et al. $2010^{\Sigma}$ & 54 & $\mathrm{NE}$ \\
\hline Devario malabaricus (Jerdon, & S1 S2 S4 S5 S6 & $1 \mathrm{C}$ & Clariidae & & \\
\hline 1849$)^{11}$ & 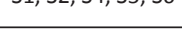 & ect & Clarias dussumieri Valenciennes, & $S_{1} \varsigma_{2}$ & NT \\
\hline D. aequipinnatus (McClelland, 1839) & S1, S3, S4, S5 & LC & 1840 ตึก & כנד וגנו & $7 w$ \\
\hline Esomus danricus (Hamilton, 1822) & S5 & LC & Heteropneustidae & & \\
\hline Rasbora dandia (Valenciennes, & $S_{1} S_{2} S_{3} S_{4} S_{5}$ & $N F_{-}-2>$ & Heteropneustes fossilis (Bloch, 1794) & $\mathrm{S} 1, \mathrm{~S} 2, \mathrm{~S} 3, \mathrm{~S} 5$ & LC \\
\hline 1844) & 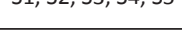 & TVL & Ambassidae & & \\
\hline $\begin{array}{l}\text { Garra menoni Indra \& Rema Devi, } \\
\text { 1984" (Image 14) }\end{array}$ & S6 & VU & $\begin{array}{l}\text { Ambassis ambassis (Lacepède, } \\
1802)^{5 / D}\end{array}$ & S5 & LC \\
\hline G. mullya (Sykes, 1839) (Image 15) & $\begin{array}{l}\mathrm{S1}, \mathrm{S} 2, \mathrm{S3}, \mathrm{S4} \\
\mathrm{S} 5, \mathrm{~S} 6\end{array}$ & LC & Parambassis dayi (Bleeker, 1874) $)^{\text {ติ }}$ & S1, S5 & LC \\
\hline G. joshuai Silas, $1954^{\text {१ा }}$ & S4, S6 & EN & P. thomassi (Day, 1870) $)^{\text {ฯा }}$ & $\mathrm{S} 1, \mathrm{~S} 2, \mathrm{~S} 3, \mathrm{~S} 4, \mathrm{~S} 5$ & LC \\
\hline Garra spa & S6 & - & Scatophagidae & & \\
\hline Balitoridae & & & $\begin{array}{l}\text { Scatophagus argus (Linnaeus, 1766) } \\
\text { s/D }\end{array}$ & S5 & LC \\
\hline $\begin{array}{l}\text { Balitora jalpalli Raghavan et al., } \\
2013^{\Sigma}\end{array}$ & S6 & NE & Hemirhamphidae & & \\
\hline Bhavania australis (Jerdon, 1849) & S4, S6 & LC & $\begin{array}{l}\text { Hyporhamphus limbatus } \\
\text { (Valenciennes, 1847) }\end{array}$ & S1, S5 & LC \\
\hline $\begin{array}{l}\text { Homaloptera menoni Shaji \& Easa, } \\
\text { 1995" }\end{array}$ & S6 & LC & $\begin{array}{l}\text { H. xanthopterus (Valenciennes, } \\
\text { 1847) }\end{array}$ & S5 & VU \\
\hline $\begin{array}{l}\text { H. pillai Indra \& Remadevi, 1981" } \\
\text { (Image 16) }\end{array}$ & S6 & LC & Belonidae & & \\
\hline Nemacheilidae & & & Xenentodon cancila (Hamilton, 1822) & S4 & LC \\
\hline $\begin{array}{l}\text { Mesonoemacheilus guentheri Day, } \\
\text { 1867 }\end{array}$ & S4, S5, S6 & LC & Aplocheilidae & & \\
\hline M. remadevii Shaji, $2002^{\Sigma}$ & S4, S6 & LC & Aplocheilus blockii Arnold, 1911 & S4, S5 & LC \\
\hline M. triangularis Day, 1865 ฯึ & S2, S4, S5 & LC & A. lineatus (Valenciennes, 1846) & S1, S2, S3, S4, S5 & LC \\
\hline Nemacheilus monilis Hora, $1921^{\text {ตा }}$ & Sc & $e_{0}$ & Syngnathidae & & \\
\hline (Image 18) & Sb & $\mathrm{LC}$ & Microphis cuncalus (Hamilton, 1822) & S5 & LC \\
\hline Schistura denisoni Day, $1867^{\text {กศ }}$ & S4, S6 & LC & Platycephalidae & & \\
\hline $\begin{array}{l}\text { S. semiarmatus Day, 1867ๆๆ (Image } \\
\text { 19) }\end{array}$ & S6 & LC & $\begin{array}{l}\text { Grammoplites scaber (Linnaeus, } \\
1758)^{5 / 0}\end{array}$ & S5 & NE \\
\hline Cobitidae & & & Centropomidae & & \\
\hline $\begin{array}{l}\text { Lepidocephalichthys thermalis } \\
\text { (Valenciennes, 1846) }\end{array}$ & $\mathrm{S} 2, \mathrm{~S} 4, \mathrm{~S} 6$ & LC & Lates calcarifer (Bloch, 1790) $)^{\mathrm{s} / \mathrm{D}}$ & S5 & $\mathrm{NE}$ \\
\hline Pangio goaensis (Tilak, 1972) & S5 & LC & Sillaginidae & & \\
\hline Bagridae & & & Sillago sihama (Forsskål, 1775) & S5 & NE \\
\hline Batasio travancoria Hora \& Law, & & & Carangidae & & \\
\hline $1941^{\prime \prime}$ & S5 & VU & $\begin{array}{l}\text { Carangoides malabaricus (Bloch \& } \\
\text { Schneider, 1801) }\end{array}$ & S5 & $\mathrm{NE}$ \\
\hline $\begin{array}{l}\text { Hemibagrus punctatus (Jerdon, } \\
\text { 1849) }\end{array}$ & S4 & CR & Leiognathidae & & \\
\hline Mystus seengtee (Sykes, 1839) & $\mathrm{S} 1, \mathrm{~S} 2, \mathrm{~S} 3, \mathrm{~S} 4, \mathrm{~S} 5$ & $\mathrm{NE}$ & Leiognathus equulus (Forsskål, & S5 & LC \\
\hline M. gulio (Hamilton, 1822) & S5 & LC & $1775)^{s /}$ & & \\
\hline M. malabaricus (Jerdon, 1849) & $\mathrm{S} 1, \mathrm{~S} 2, \mathrm{~S} 3, \mathrm{~S} 4$ & NT & Lutjanidae & & \\
\hline M. montanus (Jerdon, 1849) & S1, S2, S5 & LC & $\begin{array}{l}\text { Lutjanus argentimaculatus (Forsskål, } \\
1775)^{\mathrm{s} / \mathrm{D}}\end{array}$ & S5 & NE \\
\hline M. oculatus (Valenciennes, 1840) ฯก & S3 & LC & Gerreidae & & \\
\hline Siluridae & & & Gerres filamentosus Cuvier, $1829^{5 / D}$ & S5 & LC \\
\hline
\end{tabular}




\begin{tabular}{|c|c|c|}
\hline Family/Species & Distribution & IUCN Status \\
\hline \multicolumn{3}{|l|}{ Teraponidae } \\
\hline Terapon jarbua (Forsskål, 1775) & S5 & LC \\
\hline \multicolumn{3}{|l|}{ Nandidae } \\
\hline Nandus nandus (Hamilton, 1822) & S3, S4, S5 & LC \\
\hline Pristolepis marginata Jerdon, 1849 ทา & S4 & LC \\
\hline \multicolumn{3}{|l|}{ Cichlidae } \\
\hline Etroplus maculatus (Bloch, 1795) & $\mathrm{S} 1, \mathrm{~S} 2, \mathrm{~S} 3, \mathrm{~S} 4, \mathrm{~S} 5$ & LC \\
\hline E. suratensis (Bloch, 1790) & $\mathrm{S} 1, \mathrm{~S} 2, \mathrm{~S} 3, \mathrm{~S} 4, \mathrm{~S} 5$ & LC \\
\hline $\begin{array}{l}\text { Oreochromis mossambicus (Peters, } \\
\text { 1852) }\end{array}$ & $\mathrm{S} 1, \mathrm{~S} 2, \mathrm{~S} 3, \mathrm{~S} 4, \mathrm{~S} 5$ & - \\
\hline O. niloticus (Linnaeus, 1758) & S5 & - \\
\hline \multicolumn{3}{|l|}{ Mugilidae } \\
\hline Chelon parsia (Hamilton, 1822) (S/D $^{\text {S }}$ & S5 & $\mathrm{NE}$ \\
\hline Mugil cephalus Linnaeus, $1758^{5 / 0}$ & S5 & LC \\
\hline \multicolumn{3}{|l|}{ Eleotridae } \\
\hline Eleotris fusca (Forster, 1801) & S5 & LC \\
\hline \multicolumn{3}{|l|}{ Gobiidae } \\
\hline Glossogobius giuris (Hamilton, 1822) & $\mathrm{S} 1, \mathrm{~S} 2, \mathrm{~S} 3, \mathrm{~S} 4, \mathrm{~S} 5$ & LC \\
\hline Sicyopterus griseus (Day, 1877) & S5 & LC \\
\hline \multicolumn{3}{|l|}{ Anabantidae } \\
\hline Anabas testudineus (Bloch, 1792) & $\mathrm{S} 1, \mathrm{~S} 2, \mathrm{~S} 3, \mathrm{~S} 4, \mathrm{~S} 5$ & DD \\
\hline \multicolumn{3}{|l|}{ Osphronemidae } \\
\hline $\begin{array}{l}\text { Pseudosphromenus cupanus (Cuvier, } \\
\text { 1831) }\end{array}$ & S1, S3, S5 & LC \\
\hline $\begin{array}{l}\text { Osphronemus goramy Lacepede, } \\
1801\end{array}$ & S3 & LC \\
\hline \multicolumn{3}{|l|}{ Channidae } \\
\hline Channa marulius (Hamilton, 1822) & S5 & LC \\
\hline C. gachua (Hamilton, 1822) & S4, S6 & LC \\
\hline C. striata (Bloch, 1793) & $\mathrm{S} 1, \mathrm{~S} 2, \mathrm{~S} 3, \mathrm{~S} 4, \mathrm{~S} 5$ & LC \\
\hline \multicolumn{3}{|l|}{ Mastacembelidae } \\
\hline $\begin{array}{l}\text { Macrognathus guntheri (Day, } \\
\text { 1865) }\end{array}$ & S1, S3, S4, S5 & LC \\
\hline $\begin{array}{l}\text { Mastacembelus armatus (Lacepède, } \\
\text { 1800) }\end{array}$ & $\begin{array}{l}\text { S1, S2, S3, S4, } \\
\text { S5, S6 }\end{array}$ & LC \\
\hline \multicolumn{3}{|l|}{ Cynoglossidae } \\
\hline $\begin{array}{l}\text { Cynoglossus macrostomus Norman, } \\
1928^{5 / D}\end{array}$ & S5 & $\mathrm{NE}$ \\
\hline \multicolumn{3}{|l|}{ Soleidae } \\
\hline $\begin{array}{l}\text { Brachirus orientalis (Bloch \& } \\
\text { Schneider, 1801) }\end{array}$ & S5 & $\mathrm{NE}$ \\
\hline \multicolumn{3}{|l|}{ Tetraodontidae } \\
\hline $\begin{array}{l}\text { Carinotetraodon travancoricus (Hora } \\
\& \text { Nair, 1941) }\end{array}$ & S1, S3, S4, S5 & VU \\
\hline
\end{tabular}

S/D Secondary freshwater/diadromous;

ก" Endemic to Western Ghats; "Endemic to Kerala; 'Endemic to Bharathapuzha river system

Distribution: S1: Kalpathipuzha; S2: Chitoorpuzha; S3: Gayatripuzha;

S4: Thoothapuzha; S5: Bharathapuzha (Parali to Purathoor estuary); S6: Kunthipuzha/Silent Valley National Park

IUCN Status: CR - Critically Endangered, EN - Endangered, VU - Vulnerable, NT Near Threatened, LC - Least Concern, DD - Data Deficient, NE - Not Evaluated asee Appendix 1 for a discussion on Garra sp.

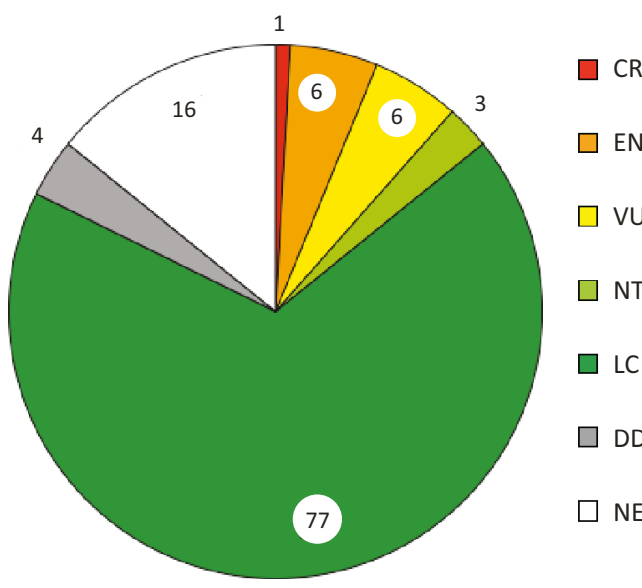

Figure 2. Conservation status of fish species occurring in Bharathapuzha River (only 113 species are listed here as $\mathbf{3}$ species were exotic to the country and the identity of 1 species needs confirmation).

includes one species listed as 'Critically Endangered' (CR) (Hemibagrus punctatus - however, see discussion in Ali et al. 2013), six species listed as 'Endangered' (EN) (however, see note on Garra joshuai) and six species listed as 'Vulnerable' (VU). Majority of species (65\%; $\mathrm{S}=77$ ) are listed as 'Least Concern' (LC). Thoothapuzha tributary had the highest number of threatened species (S=10) (Fig. 2).

\section{Fishes of the Silent Valley National Park}

The Silent Valley National Park (NP) (core area of $89.52 \mathrm{~km}^{2}$ and a buffer zone of $\left.14.70 \mathrm{~km}^{2}\right)$, and an altitude ranging from 200-2383 m) (Hosagoudar \& Riju 2013 ) is one of the most important conservation areas in the Western Ghats. Two streams, the west flowing Kunthipuzha draining the core area and the east flowing Bhavani draining the eastern segment of the buffer zone of the Silent Valley National Park (see Easa \& Basha 1995) comprise the type locality of four species, viz., Balitora jalpalli (Image 6), Garra menoni (Image 14), Homaloptera pillaii (Image 16) and Mesonoemacheilus remadevii (Image 7).

The Kunthipuzha stream of the Silent Valley National Park has been surveyed in the past by Devi \& Indra (1986) and Easa \& Basha (1995) who both recorded 11 species. During our surveys carried out in 2010, we recorded 25 species (Table 1), thereby increasing the number of freshwater fishes known from the Silent Valley NP. However, this number cannot be considered as the actual diversity of the National Park because several additional species are found in the east flowing Bhavani River and the Kadalundi River (draining the western segment of the buffer zone), which is not mentioned herein. 


\section{Range extension of Osteochilichthys longidorsalis Pethiyagoda \& Kottelat, 1994}

Osteochilichthys longidorsalis was hitherto known to be endemic to the Chalakudy and Periyar river systems where it had a very restricted distribution (see Raghavan \& Ali 2011). During recent (February 2013) field work in the Thoothapuzha tributary, we collected a single specimen (CRG-SAC.2013.01; 79.98mm SL) (Image 8) of a fish that resembled $O$. longidorsalis. Detailed examination of the specimen indicated that the measurements and counts (Table 2) were within the range of topotypic material collected from Vettilapara, Chalakudy River, and those mentioned in the original description of Pethiyagoda \& Kottelat (1994). We therefore extend the range of $O$. longidorsalis to the Bharathapuzha River system.

We believe that $O$. longidorsalis could have had a much more extensive range of distribution north and south of the Palakkad Gap in stream habitats providing a very specific niche. But over time, the continuity in the range of distribution was broken and inadequacy of geographical coverage during surveys left isolated

Table 2. Morphometric characteristics of Osteochilichthys longidorsalis collected from Bharathapuzha River

\begin{tabular}{|c|c|c|}
\hline & CRG.SAC.2013.01 & $\begin{array}{l}\text { Pethiaygoda \& } \\
\text { Kottelat (1994) }\end{array}$ \\
\hline Standard length $(\mathrm{SL})$ in $\mathrm{mm}$ & 79.9 & $102-235$ \\
\hline \multicolumn{3}{|l|}{$\% S L$} \\
\hline Total length & 136.5 & $126.8-138.1$ \\
\hline Depth of body & 30.9 & $29.2-33.2$ \\
\hline Depth of caudal peduncle & 13.2 & $11.3-13.1$ \\
\hline Length of caudal peduncle & 15.7 & na \\
\hline Lateral head length & 25.0 & $20.8-25.3$ \\
\hline Dorsal head length & 21.4 & $20.7-23.4$ \\
\hline Pre dorsal length & 48.3 & na \\
\hline Pre anal length & 75.8 & na \\
\hline Pre pelvic length & 51.2 & na \\
\hline Maximum head width & 16.1 & $14.2-16.8$ \\
\hline Maximum body width & 20.8 & $16.0-19.5$ \\
\hline Pectoral fin length & 24.4 & $22.7-26.3$ \\
\hline Pelvic fin length & 24.5 & $23.1-26.2$ \\
\hline Dorsal fin length & 28.4 & $24.1-46.6$ \\
\hline \multicolumn{3}{|l|}{$\%$ lateral HL } \\
\hline Snout length & 37.2 & $37-45$ \\
\hline Eye diameter & 31.9 & $22-29$ \\
\hline Inter orbital distance & 43.8 & $36-51$ \\
\hline Inter nares distance & 26.2 & $28-36$ \\
\hline
\end{tabular}

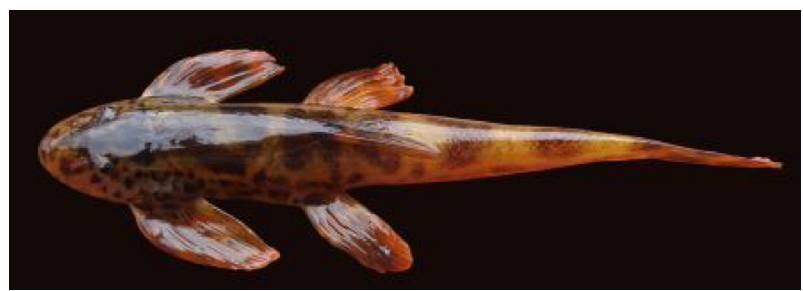

Image 6. Balitora jalpalli, a balitorid loach endemic to the Kunthipuzha stream of Silent Valley National Park [adapted from Raghavan et al. 2013b]

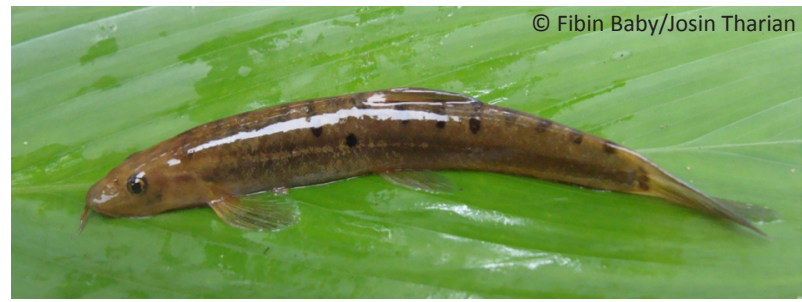

Image 7. Mesonoemacheilus remadevii, a balitorid loach endemic to the Bharathapuzha River system.

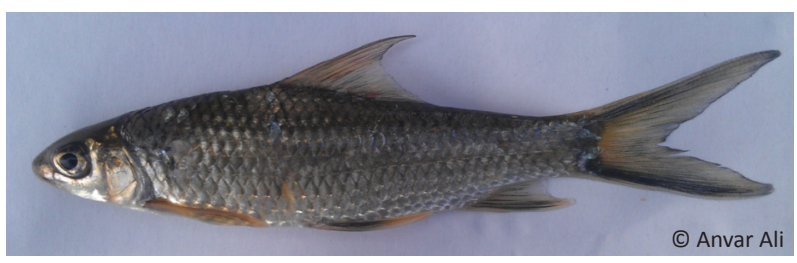

Image 8. Specimen of Osteochilichthys longidorsalis, collected from the Bharathapuzha River system.

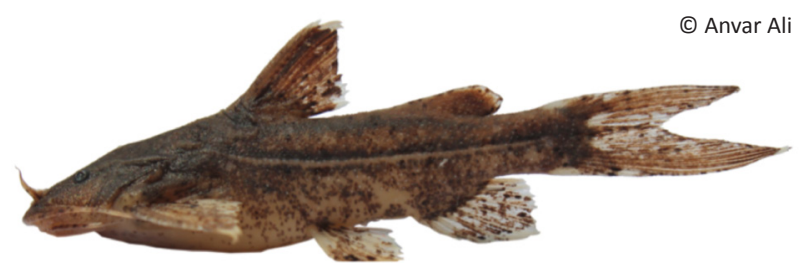

Image 9. Pseudolaguvia austrina, a sisorid catfish endemic to the Bharathapuzha River system.

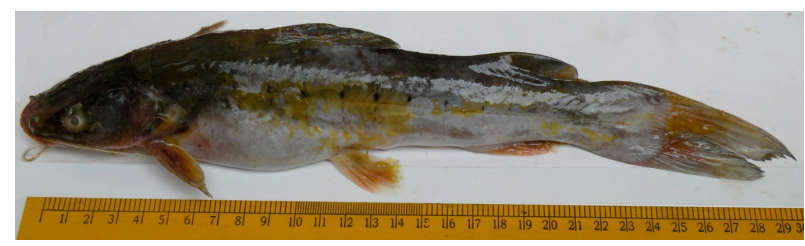

Image 10. Specimen of Hemibagrus cf punctatus recorded from Kanjirapuzha tributary of Bharatapuzha River in 2008. [Scale in $\mathrm{cm}$ ] [Adapted from Ali et al. 2013] 


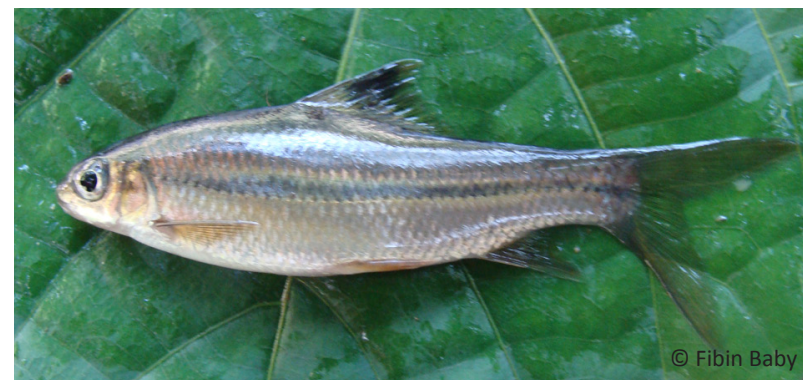

Image 11. Osteochilichthys nashii

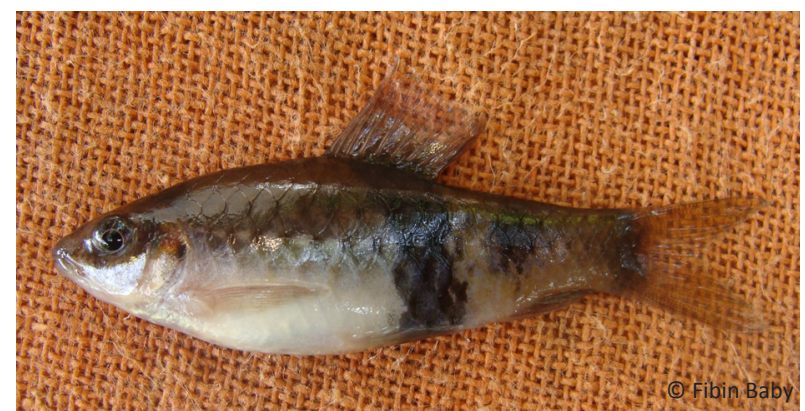

Image 13. Haludaria fasciata

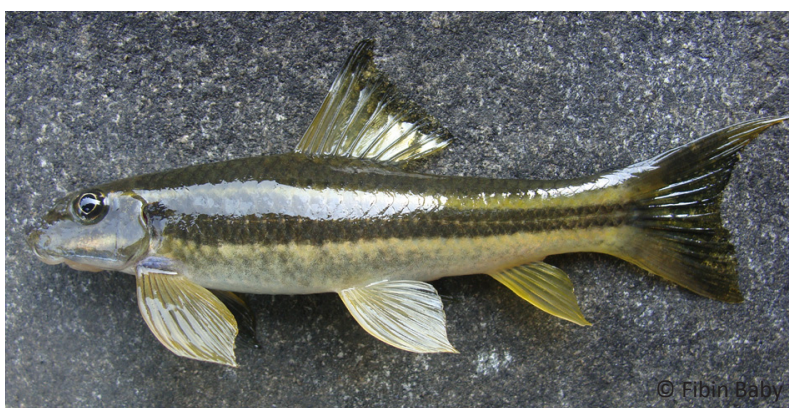

Image 15 . Garra mullya

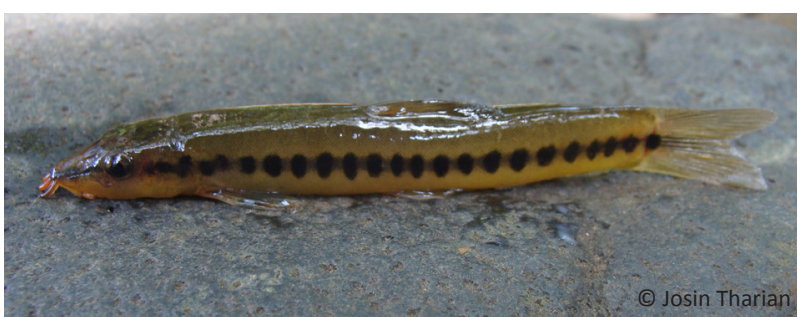

Image 18. Nemacheilus monilis

populations unreported. Thus, there is also a possibility that this species might occur in the upper reaches of the neighbouring Chaliyar basin.

We prefer to retain the generic name Osteochilichthys instead of Osteochilus as mentioned in the Catalog of Fishes (Eschmeyer 2013). The reason being that no

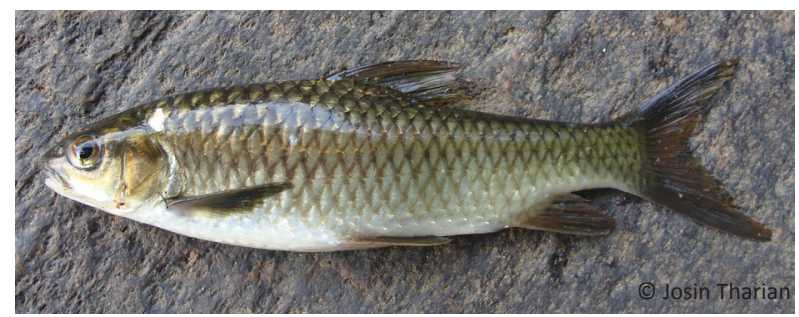

Image 12. Barbodes carnaticus

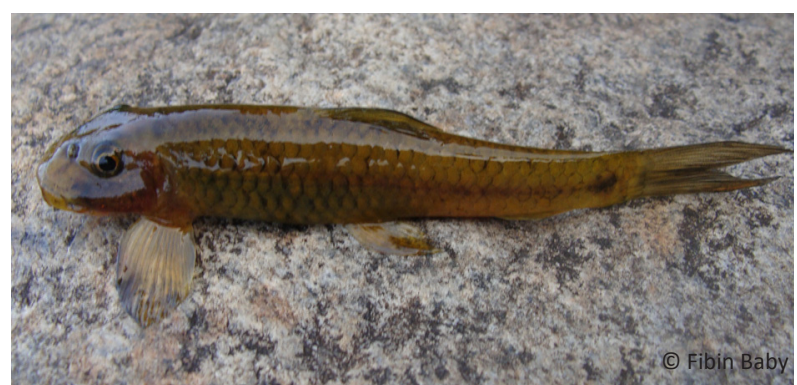

Image 14. Garra menoni

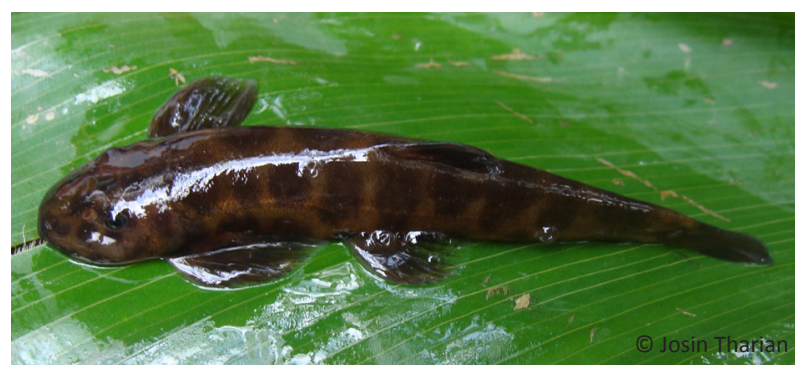

Image 16. Homaloptera pillaii

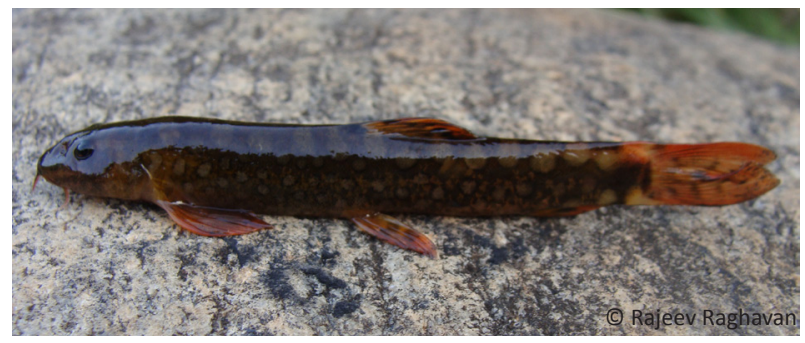

Image 17. Mesonoemacheilus guentheri

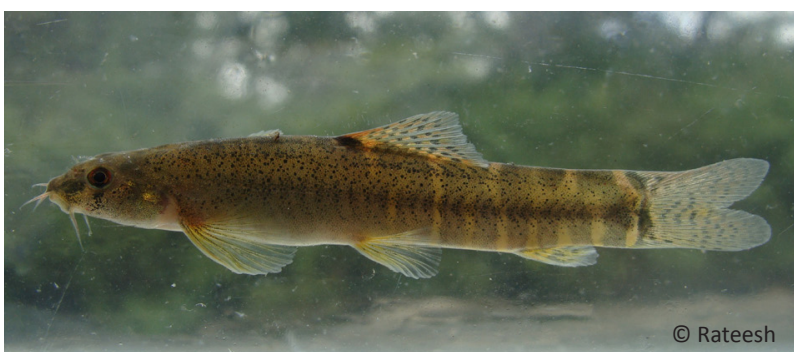

Image 19. Schistura semiarmatus 
taxonomic revision of this species has taken place and Eschmeyer (2013) merely cites Thomas et al. (2002), who, without any justification and discussion, chose to use the name Osteochilus over Osteochilichthys in their paper on the fishes of southern Kerala (see additional discussion in Appendix 1).

\section{New site record for Pseudolaguvia austrina Radhakrishnan, Suresh Kumar \& Ng, 2010}

Pseudolaguvia austrina (Image 9) was the first member of this genus described from peninsular India near the town of Mannarkad (Kunthipuzha stream) in the Bharathapuzha River system (Radhakrishnan et al. 2010). During a recent survey (February 2013), one specimen of P. austrina (CRG-SAC-2013. $11.130 .34 \mathrm{~mm} \mathrm{SL}$ ) was collected from Thoothapuzha $(\sim 20 \mathrm{~km}$ downstream of the type locality). This forms a new site record.

\section{Threats to the riverine ecosystem and biodiversity}

Bharathapuzha comprise one of the 16 catchments in the southern Western Ghats that has the highest species richness and endemism of freshwater taxa including fish, mollusc and odonates (Molur et al. 2011). It is also one of the five catchments along with Periyar, Pamba, Manimala and Chaliyar that qualify as potential freshwater 'Key Biodiversity Areas' (KBAs) (Molur et al. 2011). In spite of this, Bharathapuzha is one of the most degraded and threatened river systems in the region. Several anthropogenic stressors including deforestation and loss of riparian cover, dams and other impoundments, pollution, sand mining, non-native species, climate change and destructive fishing practices are threatening the fish diversity of Bharathapuzha River system.

Deforestation and loss of riparian vegetation: The Bharathapuzha River basin has undergone large-scale deforestation due to construction of several dams (Raj \& Azeez 2011). Deforestation is prominent in several catchment areas such as Mangalam, Nelliyampathy, Walayar, Malampuzha, Nellipuzha, Dhoni and Kalladikode. Forest lands have been transformed into largely monoculture plantations (Raj \& Azeez 2010a). During the period 1973-2005, the natural vegetation cover in the river basin declined by $31 \%$, as a result of the increase in area under plantations (Raj \& Azeez 2010b). The riparian vegetation along the Bharathapuzha and its tributaries are severely disturbed or in some cases totally destroyed. In addition, there is also a threat from invasion by exotic plants all along the river basin. The loss of forest cover at such high rates impact freshwater fishes since a significant proportion of the riverine species in the Western Ghats region exploit allochthonous food resources (Arunachalam 2000). Increased sedimentation as a result of deforestation changes the river bed habitat and thus degrades the breeding substrate of many fish species (Dahanukar et al. 2011). In this way, the deforestation in the upstream catchments of the Bharathapuzha can impact several hill stream loaches of the family Balitoridae, Cobitidae and Nemacheilidae which require pebbles and gravel in their microhabitats for breeding (Dahanukar et al. 2011). There are at least 10 species of loaches inhibiting the various hill streams tributaries of Bharathapuzha.

Dams and other impoundments: Dams are a major threat to freshwater biodiversity (Vorosmarty et al. 2010). Dams remove turbulent river sections and create tranquil water bodies, thereby affecting flow and temperature regimes, sediment transport, and species communities (Liermann et al. 2012). Several dams worldwide now impair habitat and migration opportunities for many freshwater fish species (Liermann et al. 2012) including those that are endemic and threatened (Xie et al. 2007).

Bharathapuzha has been dammed extensively, mainly for irrigation and water diversion purposes. Eleven irrigation projects and several surface dams in the river basin cater to $493.06 \mathrm{~km}^{2}$ agriculture lands (Raj \& Azeez 2010a). In addition, there are many check dams - temporary or permanent small impoundments for regulating water flow, on the Bharathapuzha. These small dams retain excess water flow during monsoon rains in a small catchment area behind the structure, thereby replenishing nearby groundwater reserves and wells. The dams and other impoundments along the Bharathapuzha River have impacted the movements of diadromous and catadromous species such as eels as evident from the lesser abundance of eels in the river in the recent past (A. Bijukumar \& R. Raghavan pers. obser. 2012). The check dams in the river are also reported to affect the water quality in upstream and downstream areas (Bijukumar \& Kurian 2008).

Pollution: Asian rivers are heavily polluted and degraded (Dudgeon 2000). Pollution has also been identified as the important threat to the fish fauna of the Western Ghats (Dahanukar et al. 2011). Bharathapuzha River basin supports extensive area under agriculture and plantations. Agro-based pollutants such as chemical fertilizers, pesticides, weedicides and nutrients are frequently washed down into the river, constituting a major ecological problem. Eutrophication has resulted in the abundance of filamentous algae and weeds in the lower reaches of the river, particularly from Chamravattom to Purakkad. In addition to agro-based 
pollutants, Bharathapuzha and its tributaries also receive substantial amount of urban sewage. For example, the town of Pattambi is one such polluted area along the river, where the urban sewage canals directly open into the river, through which the municipal waste is dumped. Such large scale pollution not only degrades the habitat but also causes endocrine disruptions and several other physiological imbalances in fish including breeding failure which could ultimately lead to their extirpation.

Limestone mining is being carried out in the catchment areas of Malampuzha in the Kalpathipuzha tributary, leading to siltation and pollution in the streams and the reservoir. Silicate content of water in this area has been found to be very high (Sushama 2003). Massive dumping of mining debris and wastes has also completely destroyed the Seemanthinipuzha, one of the streams joining the Malampuzha.

Sand mining: Over the years, indiscriminate sand mining has caused irreparable damages to several river systems on the southwestern coast of India (Sreebha \& Padmalal 2011). Indiscriminate sand mining from Bharathapuzha has contributed immensely to the destruction of the river, and is now the dominant threat to the ecosystem and biodiversity of the river basin. The entire river bed is dug up, and a large number of trucks ply through the river bed daily to collect river sand. The situation is most alarming between Pattambi and Thirunavaya, where both legal and illegal sand quarrying goes on unabatedly. We observed that in the Ottapalam Revenue Division, in addition to the 18 stations (kadavus) fixed by the Kerala Government for sand quarrying, there are several 'private' kadavus operating in parallel possibility with the connivance of authorities. The quantity of sand collected from these private kadavus exceeds those from legal ones. In addition, small-scale removal of sand by local people is also damaging the river bed in many areas. In Navalin Kadavu near the village of Peringottukurussi, sand is collected in large quantities from within the check-dams using large rafts made of rubber tubes. The sand thus collected is then loaded on to trucks and transported. In many places small-scale removal of sand is not to cater for the local demands, but for supplying the big contractors. Studies conducted by Centre for Earth Science Studies (CESS), Thiruvananthapuram (CESS 1997) have shown that the rate of sand removal from the Bharathapuzha is several times more than the natural rate of replenishment. Such massive sand removal will have a highly detrimental impact on ichthyofauna of the river as sand is the preferred breeding substrate for many fish species. In addition, sand mining alter aquatic food web as well as nutrient cycles, and is a direct threat to the survival of several species such as Glossogobius giuris and Sicyopterus griseus that prefer sand substratum.

Non native species: Six species of non-native fish occur in the Bharathapuzha (Table 1) of which three (Cyprinus carpio, Oreochromis mossambicus and $O$. niloticus) are exotic to the country, while the remaining three are the Indian major carps (Catla catla, Cirrhinus mrigala and Labeo rohita) which were transplanted from the gangetic plains for stock enhancement and aquaculture. Many reservoirs in the Bharathapuzha basin have been stocked with the non-native carps as well as the giant freshwater prawn (Macrobrachium rosenbergii) during the last several decades and have even been considered to be one of the success-stories of capture based culture fisheries (Peters \& Feustel 1998; Kutty et al. 2008). Collections of these non-native carps from the lower reaches of the river indicate their escape from the reservoirs.

The first record of the Nile Tilapia, $O$. niloticus from the rivers of Kerala was made from Bharathapuzha (Bijukumar 2008). In addition, the Mozambique Tilapia, O. mossambicus has established viable populations throughout the river, including the estuarine areas. The African catfish, Clarias gariepinus is being clandestinely cultured in many regions of the Bharathapuzha basin and may have found its way into the river system. However, we have not been able to record any specimens as yet from the wild.

Climate change: Freshwater fish are known to be at an increasing risk to climate change especially given the inextricable link between fish physiology and temperature (see Ficke et al. 2007). The Bharathapuzha watershed experiences an average annual rainfall of $2500 \mathrm{~mm}$, which is about $17 \%$ less than the state average (Anon 1998). Recent studies have observed changes in both rainfall and temperature in the river basin (Raj \& Azeez 2010a, Raj \& Azeez 2011). An overall upward trend in annual and daily temperature was observed in the river basin during 1969 to 2005 (Raj \& Azeez 2011). The impacts of climate change phenomena on the ichthyofauna of Bharathapuzha remains to be investigated further.

Unregulated aquarium fish exports: Unmanaged aquarium fish collection and exports is an emerging threat to the endemic fish diversity of Western Ghats (Raghavan et al. 2013a). In Thoothapuzha tributary, the endangered Sahyadria denisonii is being collected in massive quantities for the ornamental fish trade, even by government supported agencies such as Kerala Aquatic Ventures Private Limited (KAVIL). In addition, 
species such as Mesonoemacheilus remadevii, restricted to the Silent Valley National Park have been found to be occurring in the trade (Raghavan et al. 2013a). This shows a clear lack of co-ordination between various government departments highlighting a serious lapse in policy decisions.

Destructive fishing practices: The destructive fishing methods recorded in the river basin include use of plant poisons, dynamiting and the use of small mesh nets. Dynamiting is more prevalent in the tributaries where traditional fishermen are less in number.

\section{Conservation measures}

Like in other parts of the Western Ghats (see Dahanukar et al. 2011), the multi-stakeholder issues related to the use of fresh water in the Bharathapuzha basin has meant that indigenous fish species are least valued, and their conservation has never been a priority. Dudgeon et al. (2006) considers the protection and management of freshwater biodiversity as a conservation challenge and suggests that a combination of strategies and action plans would be highly essential to conserve freshwater ecosystems and their resources. Based on local conditions, we suggest a set of strategies that will help protect the ecosystem and facilitate the conservation and management of the native aquatic fauna of Bharathapuzha.

Integrated watershed development programs should be given top priority. To stop further ecological degradation of the river and to ensure sufficient water discharge downstream, any proposals for new check dams should be treated with caution. Similarly, we suggest that clearance should not be given to any new medium or large dam in the Bharathapuzha River basin.

Ecorestoration activities should be taken up in several stretches of the river using the River Management Fund available with district authorities. The ecorestoration activities can also be integrated into ongoing government assisted programmes such as Mahatma Gandhi National Rural Employment Generation Programme (MGNREGP) and Western Ghats Development Programme. We also suggest that all local self governments within the river basin should include ecorestoration of river as an integral component in their project planning and implementation.

As sand mining is one of the most important threats to the ecological integrity of the Bharathapuzha River system, effective enforcement mechanisms should be put into place to curb this menace. Suitable eco-friendly alternatives to sand should also be popularised by adopting awareness campaigns. Large scale cultivation and farming activities should be prohibited within the river basin, and mechanisms should be adopted to spread awareness to minimize the use of pesticides and other agro-chemicals in the plantations located in the upstream areas.

Spatial conservation options such as 'aquatic biodiversity management zones' (ABMZ) and 'fish refugias' should be declared for conserving important areas rich in endemic and threatened species. The thootha tributary is a potential site for consideration as $A B M Z$ as it harbours several endemic and threatened species, and the habitat is subjected to considerable illegal fishing including collection of endemic and threatened fishes for the aquarium trade.

There is also a need to revise the Red List status of several species of fishes including those that are endemic to the Silent Valley National Park. Many endemic species of this protected area were categorised as 'Least Concern' in view of the absence of any current or plausible future threats. However, recent studies (for e.g., Raghavan et al. 2013a) have revealed that endemic and restricted range species such as Mesonoemacheilus remadevii are being collected and exported for the aquarium pet trade thereby raising concerns on the wild populations of the other endemic balitorid and nemacheilid loaches as well.

Regulations should be brought into place to stop the unmanaged collection of endemic and threatened aquarium fishes from many areas in the river basin. Stronger enforcement is also required to prohibit the use of destructive fishing practices, especially dynamiting. Though the Ministry of Agriculture, Government of India has issued "Guidelines for the Import of Ornamental Fishes into India" based on the recommendations of the National Committee on Introduction of Exotic Aquatic Species into India, it has failed to prevent the entry of exotic fishes into the natural ecosystems of the country including the Bharathapuzha. A legally binding strategy is therefore required to regulate exotic fish into the country, and to restore the ecosystems already debilitated by the invasion of alien species.

Finally, there is a need for increased education and awareness programs to improve the conservation needs and profile of the Bharathapuzha River system. Since information on the river and its ecology is lacking, students and teachers from local schools and colleges within the river basin can be employed for data collection, monitoring and eco-restoration activities. The Biodiversity Management Committees (BMCs) at the local Panchayaths formed as per the Biological Diversity Act of India (2002), as well as the traditional 
Appendix 1: Taxonomic notes

For the sake of clarity, we provide notes on some of the generic/species names mentioned in this paper, which may be different from previous papers published from the Western Ghats/Kerala region.

Garra sp (listed in table 1). This pertains to a species of Garra that has been routinely identified in the literature as Garra gotyla stenorhynchus. Until the identity of Garra gotyla Gray is established with a neotype, and the species validity of the materials identified as Garra gotyla and G. stenorhynchus from the Western Ghats is validated, we prefer to treat the Bharathapuzha material as Garra sp.

Hypselobarbus: There is considerable taxonomic ambiguity on the generic name (Gonoproktopterus vs. Hypselobarbus) of this group. We follow Arunachalam et al. (2012) and Yang et al. (2012) and use the name Hypselobarbus instead of Gonoproktopterus.

Horalabiosa: Horalabiosa is a genus with a complex taxonomic history. For several years, Horalabiosa was considered to be a hybrid between Garra and Rasbora (See Jayaram 2010). Subsequently, the type species, H. joshuai was considered to be a synonym of Garra mullya (Talwar \& Jhingran 1991). Devi (1993) established the validity of, and re-described $H$. joshuai based on the examination of more than 500 individuals. However, recent molecular studies have re-proposed Horalabiosa as a junior synonym of Garra (Yang et al. 2012), which we follow.

Osteochilichthys: We follow Pethiyagoda et al. (2012) and Karnasuta (1993) and use the generic name Osteochilichthys (for O. longidorsalis) instead of Osteochilus as mentioned in the Catalog of Fishes (Eschmeyer 2013). The reason being that Eschmeyer (2013) merely cites Thomas et al. (2002) who without any justification and discussion, chose to use the name Osteochilus over Osteochilichthys in their paper on the fishes of southern Kerala.

Barbodes: Pethiyagoda et al. (2012) mentions that Jerdon's (1849) description of $B$. carnaticus is uninformative, and there is no known surviving type material; and as a result, subsequent authors have followed Day's (1878: 563, pl. 137) conception of the species. However, Pethiyagoda \& Kottelat (2005) suggested that the figure of Barbus carnaticus in Day (1878: pl. 137) possibly illustrates a species of Neolissochilus. However, examination of Day's materials in the Australian Museum (see Pethiyagoda et al. 2012) reveals that they differ from Neolissochilus. The exact generic status of this taxon is therefore uncertain (Pethiyagoda et al. 2012), and we retain the name Barbodes, pending detailed taxonomic investigations. Although Arunachalam et al. (2012) placed Barbodes carnaticus into another unresolved genus 'Hypselobarbus', this was not based on taxonomical evidence and/or range wide sampling. In addition, there is no mention whether they had used topotypic material of $B$. carnaticus. There are also several inconsistencies in the results of Arunachalam et al. (2012) as they illustrate a specimen of Gonoproktopterus dubius and wrongly identify it as B. carnaticus (Arunachalam et al. 2012; Fig 1. p 64).

Sahyadria: A new genus, Sahyadria has been proposed to include the two species of Redline Torpedo Barbs, Sahyadria denisonii and S. chalakkudiensis (see Raghavan et al. 2013)

fishing communities, students involved in the National Green Corps (NGC) and eco-club networks could be effectively used to monitor and conserve fish habitats. in the Bharathapuzha River basin.

\section{REFERENCES}

Ali, A., N. Dahanukar, A. Kanagavel, S. Philip \& R. Raghavan (2013). Records of the endemic and threatened catfish, Hemibagrus punctatus from the southern Western Ghats with notes on its distribution, ecology and conservation status. Journal of Threatened Taxa 5(11): 4569-4578; http://dx.doi.org/10.11609/JoTT. o3427.4569-78

Anonymous (1998). Watershed Atlas of Kerala. Vol. I and II. Kerala State Land Use Board, Kerala State Remote Sensing \& Environment Centre, Department of Space and Soil Conservation Wing.

Arunachalam, M., M. Raja, M. Muralidharan \& R.L. Mayden (2012). Phylogenetic relationships of species of Hypselobarbus (Cypriniformes: Cyprinidae): an enigmatic clade endemic to aquatic systems of India. Zootaxa 3499: 63-73.

Arunachalam, M. (2000). Assemblage structure of stream fishes in the Western Ghats. Hydrobiologia 430: 1-31; http://dx.doi. org/10.1023/A:1004080829388

Bijukumar, A. (2008). First record of Nile Tilapia, Oreochromis niloticus

L. (Pisces: Cichlidae) from Kerala. Journal of Inland Fisheries Society of India 40(2): 68-69.
Bijukumar, A. \& K.A. Abraham (2008). Impact of check dams on the hydrography of a tropical river, Bharathapuzha, Kerala, India. Екологія та ноосферологія 19(1-2): 11-18.

Bijukumar, A. \& S. Sushama (2001). The fish fauna of Bharathapuzha river, Kerala. Journal of the Bombay Natural History of India 98(3): 464-468.

Center for Earth Science Studies (CESS). (1997). A report on Bharathapuzha River. Submitted to the Government of Kerala by Centre for Earth Science Studies, Thiruvananthapuram, June, 1997.

Dahanukar, N., R. Raghavan, A. Ali, R. Abraham \& C.P. Shaji (2011). The status and distribution of freshwater fishes of the Western Ghats, pp. 21-48. In: The Status and Distribution of Freshwater Biodiversity in the Western Ghats, India. In: Molur, S., K.G. Smith., B.A. Daniel. \& W.R.T. Darwall (eds)., International Union for Conservation of Nature (IUCN), Gland, Switzerland and Zoo Outreach Organization (ZOO) Coimbatore, India.

Day, F. (1865). The Fishes of Malabar. London. i-xxxii+293pp, 20pls.

Day, F. (1878). The Fishes of India; Being a Natural History of the Fishes Known to Inhabit the Seas and Fresh Waters of India, Burma, and Ceylon Part 4. Quaritsch, London, i-xx+553-779, pls. 139-195.

Devi, K.R. (1993). Fishes of Kalakkad Wildlife Sanctuary, Tirunelveli District, Tamil Nadu, India, with a redescription of Horalabiosa joshuai Silas. Records of the Zoological Survey of India 92 (1-4): 193-209.

Devi, K.R. \& T.J. Indra (1984). Garra menoni a new Cyprinid fish from Silent Valley, Kerala, South India. Bulletin of Zoological Survey of India 5: 121-122.

Devi, K.R. \& T.J. Indra (1986). Fishes of Silent Valley. Records of Zoological Survey of India 84(1-4): 243-257. 
Devi, K.R., T.J. Indra, M.B. Raghunathan \& M.S. Ravichandran (2005) Fish fauna of the Anamalai hill ranges, Western Ghats, India. Zoos Print Journal 20(3): 1809-1811; http://dx.doi.org/10.11609/JoTT. ZPJ.1164a.1809-11

Dudgeon, D., A.H. Arthington, M.O. Gessner, Z.I. Kawabata, D.J. Knowler, C. Leveque, R.J. Naiman, A.H Prieur-Richard, D. Soto, M.L.J. Stiassny \& C.A. Sullivan (2006). Freshwater biodiversity: importance, threats, status and conservation challenges. Biological Reviews 81: 163-182; http://dx.doi.org/10.1017/ S1464793105006950

Dudgeon, D. (2000). The ecology of tropical Asian rivers and streams in relation to biodiversity conservation. Annual Reviews of Ecology and Systematics 31: 239-263; http://dx.doi.org/10.1146/annurev. ecolsys.31.1.239

Easa, P.S. \& S.C. Basha (1995). A survey of the habitat and distribution of stream fishes in the Kerala part of Nilgiri Biosphere Reserve. Kerala Fisheries Research Institute No. 104.

Easa, P.S. \& C.P. Shaji (1997). Freshwater fish diversity in Kerala part of the Nilgiri Biosphere Reserve. Current Science 73: 180-182.

Eschmeyer, W.N. (Ed). Catalog of Fishes. California Academy of Sciences (http://research.calacademy.org/research/ichthyology/ catalog/fishcatmain.asp). Electronic version accessed 21 May 2013.

Ficke, A.D., C.A. Myrick. \& L.J. Hansen (2007). Potential impacts of global climate change on freshwater fisheries. Reviews in Fish Biology and Fisheries 17(4): 581-613; http://dx.doi.org/10.1007/ s11160-007-9059-5

Herre, A.W.C.T. (1942). Glyptothorax housei, a new sisorid catfish from south India. Stanford Ichthyological Bulletin 2 (4): 117-119.

Herre, A.W.C.T. (1945). Notes on fishes in the zoological museum of Stanford University XX. New fishes from china and india - A new genus and a new Indian record. Journal of the Washington Academy of Science 35: 399-404.

Hosagoudar, V.B. \& M.C. Riju (2013). Foliicolous fungi of Silent Valley National Park, Kerala, India. Journal of Threatened Taxa 5(3): 37013788; http://dx.doi.org/10.11609/JoTT.03302.01-88

Indra, T.J. \& K.R. Devi (1981). A new species of the genus Homaloptera from Silent Valley, Kerala, South India. Journal of the Bombay Natural History Society 84: 67-70.

Jayaram, K.C. (2010). The Freshwater Fishes of the Indian Region Narendra Publishing House, New Delhi, India, 616p+XXXIX plates.

Jerdon, T.C. (1849). On the fresh-water fishes of southern India. (continued from p. 149.) Madras Journal of Literature and Science 15(2): 302-346.

Karnasuta, J. (1993). Systematic revision of southeastern Asiatic cyprinid fish genus Osteochilus with description of two new species and a new subspecies. Kasetsart University Fishery Research Bulletin 19: 105.

Kottelat, M. (2012). Conspectus cobitidium: an inventory of the loaches of the world (Teleostei: Cypriniformes: Cobitoidea). The Raffles Bulletin of Zoology Suppl 26: 1-199.

Kurup, B.M., K.V. Radhakrishnan \& T.G. Manojkumar (2004). Biodiversity status of fishes inhabiting rivers of Kerala (south India) with special reference to endemism, threats and conservation measures. In: Welcomme, R.L. \& T. Petr. (eds). Proceedings of the Second International Symposium on the Management of Large Rivers for Fisheries 2 (LARS2), Cambodia, 310pp.

Kutty, M.N., C.M. Nair \& K.R. Salin (2008). Reservoir fisheries of freshwater prawn - success story of an emerging culture-based giant freshwater prawn fishery at Malampuzha Dam in Kerala, India. Aquaculture Asia Magazine, April-June 2008: 40-41.

Liermann, C.R., C. Nilsson, J. Robertson, R.Y. Ng (2012). Implications of Dam Obstruction for Global Freshwater Fish Diversity. Bioscience 62(6): 539-548; http://dx.doi.org/10.1525/bio.2012.62.6.5

Molur, S., K.G. Smith, B.A. Daniel \& W.R.T. Darwall (compilers) (2011). The Status and Distribution of Freshwater Biodiversity in the Western Ghats. International Union for Conservation of Nature (IUCN), Gland, Switzerland and Zoo Outreach Organization (ZOO) Coimbatore, India.

Peters, D.M. \& C. Feustel (1998). Social and economic aspects of fisheries enhancements in Kerala reservoirs. In: Petr, T. (ed.). Inland Fishery Enhancements. FAO Fisheries Technical Paper 374, 463p.

Pethiyagoda, R., M. Meegaskumbura \& K. Maduwage (2012). A synopsis of the South Asian fishes referred to Puntius (Pisces: Cyprinidae). Ichthyological Exploration of Freshwaters 23(1): 69-95.

Pethiyagoda, R. \& M. Kottelat (1994). New species of fishes of the genera Osteochilichthys (Cyprinidae), Travancoria (Balitoridae) and Horabagrus (Bagridae) from the Chalakudy River, Kerala, India. Journal of South Asian Natural History 1: 97-116.

Pethiyagoda, R. \& M. Kottelat (2005).The identity of the south Indian barb Puntius mahecola (Teleostei: Cyprinidae). Raffles Bulletin of Zoology, Supplement, 12: 145-152

Radhakrishnan, K.V., S. Sureshkumar \& H.H. Ng (2010). Pseudolaguvia austrina, a new species of sisorid catfish (Osteichthyes: Siluriformes) from Peninsular India. Ichthyological Explorations of Freshwaters 21(4): 377-383.

Raghavan, R. (2011). Need for further research on the freshwater fish fauna of the Ashambu Hills landscape: a response to Abraham et al. Journal of Threatened Taxa 3(5): 1788-1791; http://dx.doi. org/10.11609/JoTT.o2755.1788-91

Raghavan, R., N. Dahanukar, M. Tlusty, A. Rhyne, K.K. Kumar, S. Molur \& A. Rosser (2013a). Uncovering an obscure trade: threatened freshwater fishes and the aquarium markets. Biological Conservation 164: 158-169; http://dx.doi.org/10.1016/j.biocon.2013.04.019.

Raghavan, R., S. Philip, A. Ali \& N. Dahanukar (2013). Sahyadria, a new genus of barbs (Teleostei: Cyprinidae) from Western Ghats of India. Journal of Threatened Taxa 5(15): 4932-4938; http://dx.doi. org/10.11609/JoTT.03673.4932-8

Raghavan, R., J. Tharian, A. Ali, S. Jadhav \& N. Dahanukar (2013b). Balitora jalpalli, a new species of stone loach (Teleostei: Cypriniformes: Balitoridae) from Silent Valley, southern Western Ghats, India. Journal of Threatened Taxa 5(5): 3921-3934; http:// dx.doi.org/10.11609/jott.03277.3921-34

Raj, P.P.N \& P.A. Azeez (2009). Spatial and temporal variation in surface water chemistry of a tropical river, the river Bharathapuzha, India. Current Science 96(2): 245-251.

Raj, P.P.N. \& P.A. Azeez (2010a). Changing rainfall in the Palakkad plains of South India. Atmosphera 23(1): 75-82.

Raj, P.P.N. \& P.A. Azeez (2010b). Land use and land cover changes in a tropical river basin: a case from Bharathapuzha River basin, southern India. Journal of Geographic Information System 2: 185193; http://dx.doi.org/10.4236/jgis.2010.24026

Raj, P.P.N. \& P.A. Azeez (2011). Temperature rise in the Bharathapuzha river basin, southern India. Current Science 101(4): 492.

Raj, P.P.N. \& P.A. Azeez (2012). morphometric analysis of a tropical medium river system: a case from Bharathapuzha River southern India. Open Journal of Modern Hydrology 2: 91-98; http://dx.doi. org/10.4236/ojmh.2012.24011

Shaji, C.P. (2002). Mesonoemacheilus remadevii (Pisces: Balitoridae. Nemeacheilinae) from Silent Valley National Park, Kerala. Indian Journal of Fisheries 49(2): 217-221.

Silas, E.G. (1958). Studies on cyprinid fishes of the oriental genus Chela Hamilton. Journal of the Bombay Natural History Society 55(1): 54-99+2pls.

Silas, E.G. (1951). On a collection of fish form the Annamalai and Nelliampathi Hill ranges (Western Ghats) with notes on its zoogeographical significances. Journal of the Bombay Natural History Society 49: 670-681.

Sreebha S. \& D. Padmalal (2011). Environmental Impact Assessment of sand mining from the small catchment river in the southwestern coast of India. Environmental Management 47: 130-140; http:// dx.doi.org/10.1007/s00267-010-9571-6

Sushama, S. (2003). Ecology and Biodiversity of Nila River. PhD Thesis, University of Kerala, India

Sushama S., R. Tresa \& A. Bijukumar (2004). Distribution of fishes in the Nila River. Indian Journal of Fisheries 51(3): 385-387.

Talwar, P. K. \& A. G. Jhingran. (1991). Inland Fishes of India and Adjacent Countries (in two volumes). Oxford \& IBH Publishing Co., New Delhi, Bombay, Calcutta. v. 1-2: i-xvii+36 unnumbered+1-1158, 
1pl, 1 map.

Thomas, K.R., M.J. George \& C.R. Biju (2002). Freshwater fishes of southern Kerala with notes on the distribution of endemic and endangered species. Journal of the Bombay Natural History Society 99 (1): 47-53.

Vorosmarty, C.J., P.B. McIntyre, M.O. Gessner, D. Dudgeon, A. Prusevich, P. Green, S. Glidden, S.E. Bunn, C.A. Sullivan, C.R. Liermann \& P.M. Davies (2010). Global threats to human water security and river biodiversity. Nature 467: 555-561; http://dx.doi.org/10.1038/nature09440

Xie, S., Z. Li, J. Liu, S. Xie, H Wang \& B.R. Murphy (2007). Fisheries of the Yangtze River Show Immediate Impacts of the Three Gorges Dam. Fisheries 32(7): 343-344.

Yang, L., M. V. Hirt, T. Sado, M. Arunachalam, R. Manickam, K.L. Tang, A.M. Simons, H.-H. Wu, R.L. Mayden \& M. Miya (2012). Phylogenetic placements of the barbin genera Discherodontus, Chagunius, and Hypselobarbus in the subfamily Cyprininae (Teleostei: Cypriniformes) and their relationships with other barbins. Zootaxa 3586: 26-40.
Author Details: A. BIJUKUMAR is interested in biodiversity documentation and taxonomy, and involved in biodiversity/ environmental education activities to facilitate conservation. SIBY PHILIP is interested in molecular phylogenetics, evolution and biogeography of freshwater fishes of the South Asia region. ANVAR ALI interested in taxonomy and systematics of freshwater fishes of the Western Ghats.

S. SUSHAMA is interested in ecology of freshwater systems. RAJEEV RAGHAVAN is interested in interdisciplinary research focused on generating information and developing methods to support conservation decision-making freshwater ecosystems. 